\title{
Double Dissociation of Exteroceptive and Interoceptive Feedback Systems in the Orbital and Ventromedial Prefrontal Cortex of Humans
}

\author{
Elisabeth Hurliman, Jennifer C. Nagode, and José V. Pardo \\ Cognitive Neuroimaging Unit, Veterans Affairs Medical Center, Minneapolis, Minnesota 55417, and Departments of Psychiatry and Psychology, University \\ of Minnesota, Minneapolis, Minnesota 55455
}

The primate ventral prefrontal cortex contains two densely interconnected subregions: a lateral/orbital cortex processing primarily sensory/exteroceptive information, and a ventromedial cortex processing primarily visceroappetitive/interoceptive information. These regions have major afferents from and efferents to other associative cortices. The organization of these structures leads to an hypothesized role in feedback processing. We use neuroimaging to test this model, defined so far mostly through anatomical studies.

Healthy volunteers were trained operantly on a transitive inference task $(A>B, B>C \Rightarrow A>C)$ requiring flexible manipulation of feedback to solve. Two groups of subjects learned an arbitrary face hierarchy, one adjacent face pair at a time; each group received either visual/exteroceptive ("XXXX") or visceroappetitive/interoceptive (fruit juice) feedback for correct responses to adjacent face pairs. After task acquisition, the subjects were tested on novel stimulus pairs (i.e., nonadjacent, TEST) derived from the acquired hierarchy. The TEST condition required transitive inference. No feedback was provided during TEST.

Brain activity during TEST in the group trained with visual/exteroceptive feedback increased in the orbital prefrontal cortex and decreased in the ventromedial prefrontal cortex. In contrast, brain activity during TEST in the group trained with visceroappetitive/ interoceptive feedback decreased in the orbital prefrontal cortex and increased in the ventromedial prefrontal cortex.

These results provide functional evidence, consistent with previous anatomical studies, for two major feedback systems in human ventral prefrontal cortex: a lateral system specialized for exteroceptive information and a medial system specialized for interoceptive information. Although highly interconnected, there is a double dissociation of function between these networks in healthy humans.

Key words: feedback processing; ventral prefrontal cortex; PET neuroimaging; transitive inference; associative learning; double dissociation

\section{Introduction}

The ventral prefrontal cortex (VPFC) participates in diverse cognitive functions, including representation of primary and secondary sensory information, associative learning, flexible updating of learned associations, and sensory-specific satiety (for review, see Rolls, 1996). It also seems critically involved in decision making and goal-directed behavior (Bechara et al., 1997; Elliott et al., 2000; Winstanley et al., 2004). A common theme across the cognitive functions of this region is their dependence on feedback.

Anatomical data indicate that the primate VPFC functions in feedback processing. Barbas and Rempel-Clower (1997) found

Received June 28, 2004; revised March 4, 2005; accepted March 22, 2005.

This work was supported in part by the Department of Veterans Affairs (J.V.P., E.H.), the National Alliance for Research on Schizophrenia and Depression (J.V.P.), National Institutes of Health Grants 5T32GM08244 and 2T32GM08471-05 (J.C.N.), the American Legion (J.C.N.), and the American Legion Auxiliary Brain Sciences Center (J.C.N.). We thank our volunteers for their generosity and patience. We also thank our research team members at the Cognitive Neuroimaging Unit, in particular Joel T. Lee, Matthew C. Hagen, and Dorothy Mattson.

Correspondence should be addressed to Dr. José V. Pardo, Cognitive Neuroimaging Unit, Veterans Affairs Medical Center, One Veterans Drive, Minneapolis, MN 55417. E-mail: pard0001@umn.edu.

DOI:10.1523/JNEUROSCI.2563-04.2005

Copyright $\odot 2005$ Society for Neuroscience $\quad$ 0270-6474/05/254641-08\$15.00/0 that the VPFC projects to the more laminated dorsal prefrontal cortex in a feedback pattern. In turn, dorsolateral prefrontal regions project to ventral regions in a feedforward pattern. Öngür and Price (2000) and Öngür et al. (2003) proposed that two distinct, yet interacting, networks exist within VPFC: a lateral sensory network processing exteroceptive information [orbital prefrontal cortex (OPFC)] and a medial visceral network [ventromedial prefrontal cortex, (VMPFC)] processing interoceptive information such as visceromotor output.

Compared with the broad literature concerning exteroceptive/sensory systems, less is known about interoception. Interoception is the sense of the physiological condition of the body including, but not limited to, the viscera (for review, see Craig, 2002). Small-diameter afferents pass information of the physiological status of tissues on to higher cortex. Interoception subsumes multiple processes, such as nociception, thermoregulation, $\mathrm{pH}$ balance, blood pressure, thirst, swallowing, skeletal muscle stretch, neuroimmune interactions, etc. We previously noted large cortical activations when attempting to taste deionized, distilled water reflecting both intraoral somatosensory processing as well as fluid stimulation of the tongue and oropharynx (Zald and Pardo, 2000b). Interoceptive information inherently 
has an affective component that provides motivation; likewise, water is a primary, unconditioned reinforcer. Although the administration of fluids has a chemoreceptive component attributable to taste, the value of such a stimulus directly depends on the physiological needs of the body and has relevance for maintenance of homeostasis and survival; these latter aspects group such stimuli within interoception (Craig, 2002).

In the neuroimaging literature, the term "feedback" is used loosely to refer to information after a response. However, in the present study, feedback is defined more narrowly as information provided after a response critical for responding correctly during incremental/operant learning. Feedback, whether exteroceptive or interoceptive, is processed through circuits that decode information about the feedback stimulus as well as assign relative value to the response.

Here, we probe the functional aspects of the anatomically hypothesized VPFC feedback systems in humans using ${ }^{15} \mathrm{O}$-water positron emission tomography (PET) and a feedback-dependent cognitive activation task. We expect to see OPFC activations in the exteroceptive feedback condition; we expect to see VMPFC activations in the interoceptive feedback condition. In addition, we are interested in exploring the functional nature of interaction between the two subsystems. In a serial model, exteroceptive sensory input reaches the OPFC interacting with the interconnected VMPFC to produce visceromotor output: both networks should activate. In a parallel model, under constraints of limited capacity for dual processing, reciprocal activations should occur (i.e., lateral activations would occur with medial deactivations and vice versa).

\section{Materials and Methods}

\section{Experiment 1: visual feedback during training}

Subjects. Ten subjects (mean age, 23 years; age range, 19-29 years; three women) without significant medical history were recruited from the local University of Minnesota campus. All were free of lifetime occurrence of major psychiatric disorders [per computerized Diagnostic Interview Schedule (DIS) screener] (Bucholz et al., 1991). Seven subjects were right-handed, two subjects were ambidextrous, and one subject was left-handed [Edinburgh Handedness Inventory (Oldfield, 1971)]. All subjects gave written informed consent as approved by the Institutional Review Committee and the Radioactive Drug Research Committee.

Cognitive activation task. For an initial description of this task, please refer to the work of Nagode and Pardo (2002). We designed a feedbackdependent cognitive activation task based on transitive inference (TI) $(A>B, B>C \Rightarrow A>C)$, with pictures of 10 faces arranged hierarchically along an arbitrary dimension (Nagode and Pardo, 2002). The underlying hierarchy was initially created at random but remained constant during each subject's training, as well as across subjects. Two hierarchies of male faces and two hierarchies of female faces were created. Stimulus hierarchy was randomly assigned across subjects of a given gender. During training outside of the scanner, two adjacent faces of the hierarchy were displayed on a video screen $(\sim 50 \mathrm{~cm}$ from the subject) with the program MEL 2.0 (Psychology Software Tools, Pittsburgh, PA). The faces were $\sim 7 \times 13 \mathrm{~cm}$ black and white images presented in pairs. Subjects selected the face higher on the underlying hierarchy. Responses were made by pushing one of two keys on a response box within $3 \mathrm{~s}$ of face presentation (right response key pushed by right index finger indicates choice of right face as higher on hierarchy; left response key pushed by left index finger indicates choice of left face as higher on hierarchy). The first response for each face pair required a random guess, but future responses could rely on feedback for the face pair received previously. Trialwise feedback was provided in the visual modality ("XXXXX" appears under the correct face). Training continued until criterion ( $90 \%$ correct responses). The subjects learned the relationship $(>)$ between the two faces in an adjacent pair incrementally based on feedback. For instance, one stimulus presentation during training could be face 2 presented on the left half of the monitor and face 3 presented on the right half. No nonadjacent faces in the hierarchy are presented during training (e.g., subjects during this portion of the task will never see face 2 together with face $4,5,6$, etc.). At the end of training, once the entire hierarchy has been successfully acquired, this task is no longer feedback dependent.

During neuroimaging, subjects solve the adjacent pairs condition as a CONTROL condition (e.g., presentation of face 2 and 3 , face 3 and 4 , etc., from the underlying hierarchy of 10 faces) and a nonadjacent pairs condition (TEST) as experimental condition. During TEST, subjects solve the task by identifying in the absence of feedback the relationship between faces in novel pairs generated from nonadjacent faces from the hierarchy (TEST; e.g., presentation of face 2 on one half of the screen together with face 4 on the other half of the screen, 2 and 5, 2 and 6, etc., but never 2 and 3 or any other adjacent faces from the underlying hierarchy). In TEST, subjects were asked to select the correct one of two nonadjacent faces generated at random. The TEST pairs were allowed to differ in the degree of separation (i.e., symbolic distance) in the underlying hierarchy. First and last faces of the hierarchy were not used. No feedback was provided during TEST. Because TEST contains no feedback, the only way to respond correctly is through inference based on feedback received during CONTROL (adjacent faces).

Selection of the adjacent pairs CONTROL condition was based on several factors. First, this task offers the needed comparison condition for experimental activation tasks that tap the VPFC. This focus on comparison conditions is needed because the VPFC does not lend itself to an eyes-closed rest comparison because of its high tonic resting activity. Second, this task mimics closely the cognitive demands from the TEST condition yet is much less dependent on using previously received feedback to solve it successfully. Although CONTROL provides a close match to the cognitive demands of the TEST condition, this match is not absolute. There is a small difference in demands to working memory defined more broadly than just keeping information on-line insofar as TEST requires an inference judgment, whereas CONTROL does not. However, a previous study from our laboratory, which directly manipulated visual working memory for faces, did not find activations of interest identified in the current set of experiments. In the working memory study, a direct comparison was made between memory for faces (high working memory demands) to a control task of scrambled faces (few if any working memory demands) (Kuskowski and Pardo, 1999). Similarly, Rushworth et al. (1997) did not find that primates with inferior convexity lesions (i.e., removal of lateral area 47/12) had any significant decrements on a visual working memory task.

Overall, the TI paradigm under study has several advantages. It is based on a widely used behavior paradigm, i.e., incremental, associative learning, which warrants additional study. It permits the use of several types of feedback (e.g., cognitive, appetitive). Performance of the TEST task is not stimulus bound, i.e., correct performance requires the use of previous feedback; thus, sensory confounds from actual feedback administration can be controlled. The paradigm contains a feedbackindependent comparison condition (adjacent faces) once the task is well learned. This is of particular importance for studying VPFC function because this region has high tonic activity at rest. Thus, comparison with a resting state will obscure task-dependent VPFC activation. The paradigm provides a route around the use of resting brain states.

PET scanning. Images were acquired using an ECAT 953B scanner (Siemens, Knoxville, TN) in three-dimensional mode with septae retracted. A two-dimensional transmission scan was used for attenuation correction. $\mathrm{H}_{2}{ }^{15} 0$ PET was used to measure whole-brain activation (31 slices, $3.5 \mathrm{~mm}$ slice separation) with uniform signal recovery. A catheter provided venous access for tracer injection. A vacuum-molded pillow was used for head stabilization. Slow-bolus ( $30 \mathrm{~s}$ ) intravenous injections of $\mathrm{H}_{2}{ }^{15} 0$ (9.25 MBq or $0.25 \mathrm{mCi} / \mathrm{kg}$ initially) were administered at the beginning of each trial. Acquisition scans ( $90 \mathrm{~s}$ ) for each of the following conditions were collected: resting with eyes closed; performing a sensorimotor control task (random button press while viewing unfamiliar face pairs); selecting the correct face from an adjacent face pair (CONTROL); and selecting the correct face from a nonadjacent face pair (TEST). No feedback was provided during any of these trials. Each condition was followed by a $10 \mathrm{~min}$ interscan interval to allow isotope decay. 
Data analysis. In-house software was used to align the transmission scan and emission scans. Images were reconstructed with filtered back projection using a Hanning filter. Final image resolution was $9 \mathrm{~mm}$ fullwidth half-maximum. Estimated regional cerebral blood flow (rCBF) is based on normalized tissue radioactivity to 1000 counts. Automated software was used to assess motion artifacts, and scans were excluded if motion exceeded $2 \mathrm{~mm}$ or $2^{\circ}$ during a scan session (Minoshima et al., 1994). Nonlinear warping normalized the anatomy to a standard stereotactic atlas (Talairach and Tournoux, 1988). Scans from each condition were averaged across subjects for pixelwise group analysis. Significant activations were defined as those equal or exceeding a $Z$ value of $3.1(p<$ 0.001 ). In addition, individual subtraction images were obtained for region-of-interest analysis. Figures of functional images were produced with in-house software [iiv (Lee and Pardo, 2000)] and BrainTools software (mri3dX; K. Singh, Aston University, Birmingham, UK; http://www.aston.ac.uk/lhs/staff/singhkd/mri3dX/mri3dX.jsp).

\section{Experiment 2: interoceptive feedback during training}

Subjects. Seventeen subjects were recruited initially from the local university community and gave written informed consent. Three subjects were excluded because of equipment malfunction. One subject was excluded because of severe motion artifacts. Two subjects were excluded because of failure to reach the learning criterion of $90 \%$ correct. The final sample consisted of 11 subjects (four female), with a mean age of 26 years (range, 19-45 years). This final sample was closely matched to our sample from experiment 1 on demographic variables, including age, sex, and race. All were free of lifetime prevalence of major psychiatric disorders [per computerized DIS screener (Bucholz et al., 1991)]. None had major medical illness. Ten subjects were right-handed, and one was lefthanded. No subject had participated in experiment 1 with visual/exteroceptive feedback. To control for satiety effects, subjects who arrived at their training session more than $2 \mathrm{~h}$ after their last meal were provided a snack. No subject felt hungry or thirsty at the start of training.

Cognitive activation task. Training on TI with visceroappetitive/interoceptive feedback occurred until performance reached $90 \%$ correct. Fruit juice was selected for feedback based on subject palatability. An automated pump system controlled by MEL 2.0 software (Psychology Software Tools) delivered $0.25 \mathrm{ml}$ of fruit juice via a plastic tube into the subject's mouth for a correct response. Fruit juice was selected as the interoceptive feedback stimulus because of its extensive oropharyngeal processing beyond sensorimotor information (salivation, temperature, and viscosity), its ease of implementation in operant training, and its strong motivational component. To ensure that the juice was perceived as palatable, subjects reported any decrease in palatability during rest breaks every $10 \mathrm{~min}$. The juice was changed according to the subject's preference. We note that this interoceptive feedback condition differs from the exteroceptive feedback condition in terms of reward properties. However, interoceptive feedback processing has an inherent hedonic component that rules out any attempt to stimulate interoceptive feedback processing independently of rewarding (either pleasant or aversive) properties of the stimulus (for review, see Craig, 2002). The reward properties were minimized by using satiated subjects. Also, the repeated administration of the same fruit juice likely led to habituation in primary taste cortex (no anterior insula activation occurred during training with fruit juice), as well as sensory-specific satiety.

The CONTROL condition was matched in every way to the TEST condition, except the stimulus pairs were adjacent and had been acquired previously. Of note, CONTROL, like TEST, had no feedback. In addition to the advantages of the CONTROL condition reported for experiment 1 , this comparison (TEST - CONTROL) avoids the confounding effects of visceral sensory processing in frontal secondary association cortices. As mentioned for experiment 1 , one potential cognitive difference beyond feedback processing is the slightly increased demands on working memory in the TEST condition because of the required inference judgments. However, in this set of experiments, we included a sensorimotor control condition. The comparison (TEST - SENSORIMOTOR CONTROL) did not show significantly more active medial VPFC in the TEST condition, which has significantly greater working memory demands than the
SENSORIMOTOR CONTROL of passively viewing faces while making random button pushes.

PET scanning and data analysis paralleled the procedures described for experiment 1 .

\section{Results}

\section{Behavioral results}

Mean correct performance in experiment 1 for TEST was $98 \%$ and for CONTROL was $96 \%$. Mean correct performance in experiment 2 for TEST was $95 \%$ and for CONTROL was $93 \%$. Percentage correct responses were transformed to arcsine values to achieve a normal distribution. Independent sample $t$ tests showed significant differences between TEST - CONTROL across feedback groups $\left(t_{(19)}=2.37 ; p=0.029\right)$. The difference may reflect technical differences in training necessitated by the different feedback conditions. Nevertheless, both feedback groups performed above our performance criterion of $90 \%$ correct. More importantly, performance during TEST was not significantly different across feedback groups $\left(t_{(18)}=0.462 ; p=\right.$ $0.650)$.

\section{Whole-brain pixelwise subtractions: reciprocal lateral and medial VPFC activations}

Figure 1 demonstrates the reciprocal changes that occur in OPFC and VMPFC during the two feedback conditions. OPFC regions significantly activate in the contrast TEST - CONTROL for the visual/exteroceptive feedback condition (Fig. 1, Table 1). These networks arise bilaterally. In contrast, a trend toward OPFC deactivation emerged for the visceroappetitive/interoceptive feedback condition (coordinates, $39 \mathrm{~mm}, 35 \mathrm{~mm},-9 \mathrm{~mm} ; Z$ score of -2.6). The VMPFC deactivates for the visual/exteroceptive feedback condition (Table 2) and activates for the visceroappetitive/ interoceptive condition (Fig. 1). Of note, one of the highly deactivated VPFC regions in the visual/exteroceptive feedback condition coincides closely in location with a region highly activated in the visceroappetitive/interoceptive feedback condition. Thus, medial and lateral VPFC activity found in whole-brain group analyses followed a reciprocal pattern across external and internal feedback modalities.

In contrast, in the visceroappetitive/interoceptive feedback condition, VMPFC was activated, not OPFC (Table 3, Fig. 2). Additional significant medial activations were found for the visceroappetitive/interoceptive feedback condition in the parahippocampal-amygdaloid transitional area and ventral tegmental area/substantia nigra (VTA/SN) (Table 3, Fig. 2). Note that the anatomical labels and Brodmann areas (BA) should be considered approximate because of inherent limitations in resolution associated with PET imaging.

Although the observed changes in activity in the visceroappetitive/interoceptive feedback condition generally were lower than those in the visual/exteroceptive feedback condition, activity changes in the VPFC followed our initial hypotheses in location and pattern of activation. In addition, despite the slightly lower magnitude of activity changes in the visceroappetitive/interoceptive feedback condition, activations and deactivations were highly significant when considering resolution elements in our predefined VPFC region of interest.

\section{Region-of-interest analysis: lateral OPFC, medial VPFC, and amygdala}

Additional evidence for the double-dissociated interaction between the two ventral prefrontal systems emerges in a repeated measures ANOVA between the two feedback types (visual/ex- 
teroceptive and visceroappetitive/interoceptive) and three regions of interest (VMPFC, OPFC, and amygdala) (Fig. 3). Peak pixel activations based on the actual data set were used to delimitate regions of interest. We selected the amygdala as an additional region of interest because of its well established, dense neural connections with ventral prefrontal areas (Porrino et al., 1981). The VMPFC and amygdala change activity together. Mean activity in the exteroceptive feedback condition shows a decrease in both amygdala and VMPFC regions and an increase in the OPFC region. The opposite pattern emerges in the interoceptive feedback condition, because the amygdala and VMPFC regions show increased $\mathrm{rCBF}$ and the OPFC exhibits decreased rCBF (Fig. 3, Table 4).

In addition to the data-driven regionof-interest analysis, a global brain analysis was conducted in SPM99 (Wellcome Department of Cognitive Neurology, London, UK) for interactions between feedback conditions and the ventral prefrontal regions of interest. Results indicate significantly medial VPFC activation in the interoceptive feedback condition ( $Z$ score of $2.5)$, and significant OPFC activation was found in the exteroceptive feedback condition ( $Z$ score of 2.7) (Figs. 4, 5).

\section{Discussion}

\section{Overview of results}

Results of both experiments suggest the VPFC contains two feedback-processing systems, consistent with hypotheses derived from anatomical studies (Barbas and Rempel-Clower, 1997; Öngür et al., 2003). One subsystem, situated laterally in the VPFC, preferentially processes information from the external environment; the other subsystem, situated medially, preferentially processes information from the internal/bodily environment.

In addition, our data reveal that the two feedback systems function in a double-dissociated manner. Inference based on review of visual/exteroceptive feedback history activates OPFC and deactivates VMPFC. Conversely, inference based on review of visceroappetitive/interoceptive feedback history activates VMPFC and deactivates OPFC. The two VPFC systems thus seem poised toward competition.

Considerably less afferent sensory input exists to VMPFC than to OPFC. Taste feedback could activate OPFC regions such as posterolateral orbital cortex, and VMPFC regions may primarily process monitor visceromotor output and participate in decision making in this manner (Öngür and Price, 2000). Here, a functional double dissociation between VMPFC and OPFC would necessitate an inhibitory feedback loop. Visceral information travels via spinal, facial, glossopharyngeal, and vagal afferents before reaching the brainstem and cortex (Cechetto and Chen, 1990). Fine afferents carrying interoceptive information reach hypothalamus and periaqueductal gray (Craig, 2002), which in turn are connected to VMPFC and limbic regions. Via reciprocal connections from amygdala to VMPFC (Price, 2003), interoceptive information may reach VMPFC without traveling through
Table 1. Significant activations in the lateral ventral prefrontal cortex (OPFC) during TEST - CONTROL, exteroceptive feedback during training

\begin{tabular}{llllll}
\hline & & \multicolumn{3}{l}{ Coordinates } & \\
\cline { 3 - 5 } Region & BA & $x$ & $y$ & $z$ & Zscore \\
\hline Inferior frontal gyrus (POG) & 111 & -33 & 30 & -18 & 4.9 \\
Middle frontal gyrus (TOS) & $47 / 12 r$ & 39 & 37 & -16 & 4.5 \\
Middle frontal gyrus (AOG) & 100 & 35 & 50 & -9 & 4.3 \\
Middle frontal gyrus (AOG) & 111 & 28 & 39 & -18 & 4.3 \\
Superior frontal gyrus (AOG) & 111 & 28 & 44 & -16 & 4.3 \\
Superior frontal gyrus (AOG) & 100 & -30 & 53 & -14 & 3.6 \\
\hline
\end{tabular}

Regions in parentheses are per the three-dimensional probabilistic atlas (Chiavaras et al., 2001). AOG, Anterior orbital gyrus; POG, posterior orbital gyrus; TOS, transverse orbital sulcus. Brodmann areas (BAs) are assigned per Price (Öngür et al., 2003), mapped onto the atlas of Talairach and Tournoux (1988).

Table 2. Significant deactivations in the lateral ventral prefrontal cortex (OPFC) during TEST - CONTROL, exteroceptive feedback during training

\begin{tabular}{llllll}
\hline \multirow{2}{*}{ Region } & & \multicolumn{3}{c}{ Coordinates } & \\
\cline { 4 - 6 } & BA & $x$ & $y$ & $Z$ & Z score \\
\hline Gyrus rectus (GR) & $14 \mathrm{r}$ & 1 & 21 & -18 & -3.9 \\
Medial frontal gyrus & $11 \mathrm{~m}$ & -6 & 32 & -14 & -3.2 \\
\hline
\end{tabular}

Regions in parentheses are per the three-dimensional probabilistic atlas (Chiavaras et al., 2001). Brodmann areas (BAs) are assigned per Price (Öngür et al., 2003) mapped onto the atlas of Talairach and Tournoux (1988).

OPFC first. Although the number of afferents for exteroception far outnumbers those for interoception, visceral information is not passed on in an undifferentiated manner (Jänig, 1996; Vaitl, 1996).

The current study presents novel findings regarding two double-dissociated VPFC feedback systems. Previous studies examined different functions of VPFC subregions with one feedback type. O'Doherty et al. (2000) studied monetary feedback. Although money is an external stimulus, it is a potent reinforcer 
Table 3. Significant ventromedial activations (VMPFC) in TEST - CONTROL, interoceptive feedback during training

\begin{tabular}{lcccccc}
\hline & & \multicolumn{3}{c}{ Coordinates } & \\
\cline { 4 - 6 } Region & BA & $x$ & $y$ & $z$ & Zscore \\
\hline Extended amygdala & 34 & 10 & -6 & -11 & 3.6 \\
Ventral tegmental area/substantia nigra & & 10 & -19 & -9 & 3.3 \\
Gyrus rectus (GR) & $14 \mathrm{r}$ & 6 & 21 & -16 & 3.1 \\
\hline
\end{tabular}

The region in parentheses is per the three-dimensional probabilistic atlas (Chiavaras et al., 2001). Brodmann areas (BAs) are assigned per Price (Öngür et al., 2003), mapped onto the atlas of Talairach and Tournoux (1988).

because it becomes closely associated with food/shelter, comfort, and pleasure. The feedback systems operating on monetary rewards and punishments require additional dissection.

\section{Experimental issues}

The selection of the comparison TEST - CONTROL was motivated by several factors. First, high tonic resting activity of the VPFC required a nonresting comparison task (Gusnard and Raichle, 2001; Mazoyer et al., 2001). Second, studying feedback processing without confounded sensory aspects requires a feedback stimulus-independent task during scanning. During TEST, no feedback is administered, yet only with review of previously received feedback during training can the TEST condition be solved. CONTROL during PET scanning (i.e., after a long training phase) is overlearned and feedback independent.

Working memory demands differ across TEST and CONTROL, but this does not explain the current activations. When examining another comparison (TEST - SENSORIMOTOR

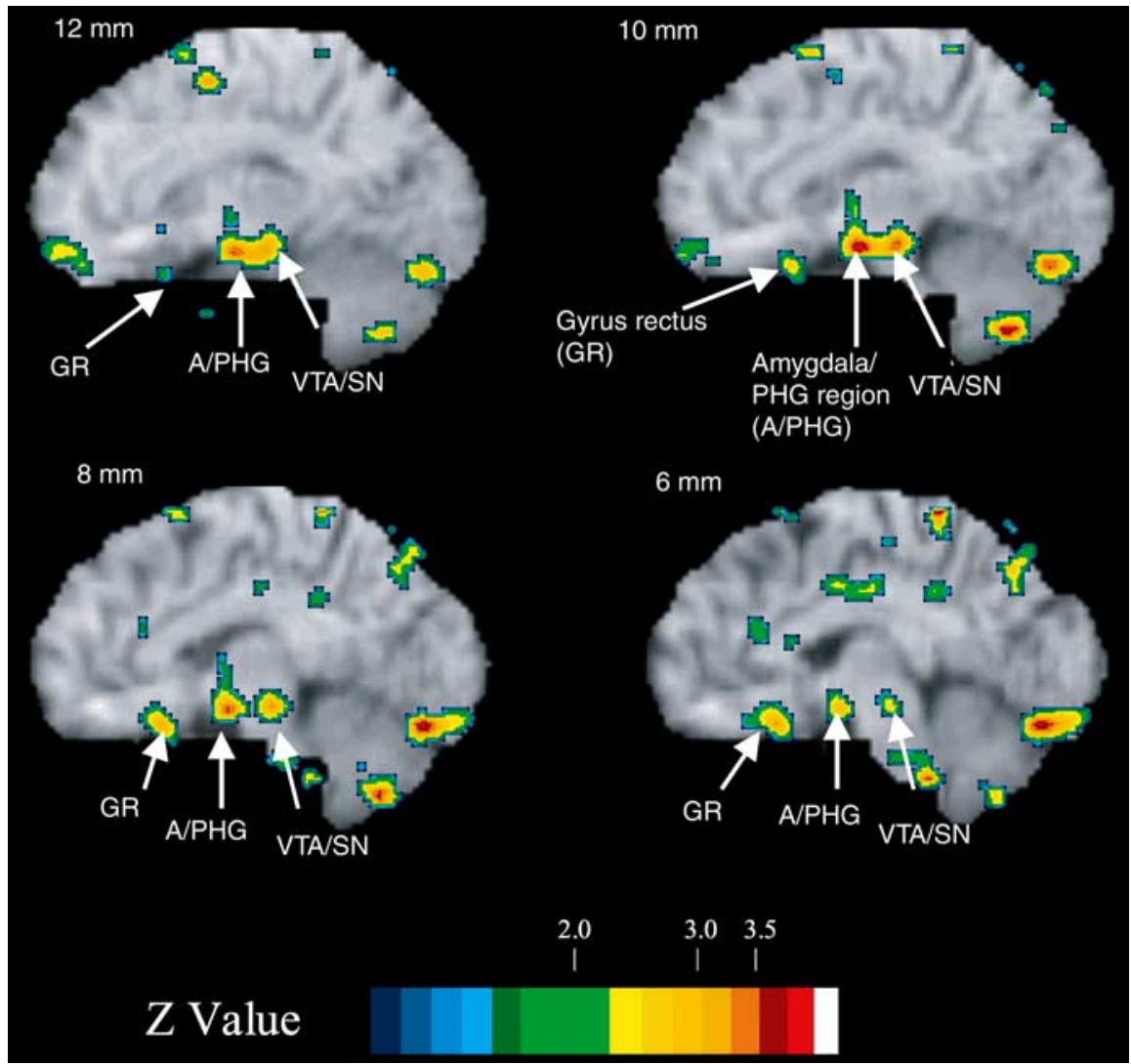

Figure 2. Sagittal slices for neural activations in the contrast between TEST and CONTROL under interoceptive feedback during training. Changes in activity are overlaid on sagittal slices of a standard brain MRI. Color scales show activations $(+)$ and deactivations (-) in Z scores. Slices are shown in millimeters. Note that TEST requires inference based on review of the feedback history without feedback stimulation; feedback stimulation occurred only during training. PHG, Parahippocampal gyrus.
CONTROL, i.e., few working memory demands during passive viewing of faces and random button pushes) for the interoceptive feedback group, no VMPFC activations emerge. This result is consistent with Rushworth et al. (1997) and Kuskowski and Pardo (1999), who have used a direct visual working memory task using face stimuli.

We implemented several experimental controls to keep constant rewarding properties of fruit juice feedback. First, we attempted to ensure that volunteers were satiated before task acquisition. Second, we adjusted the fruit juice per individual preference throughout training as needed. Third, we did not provide interoceptive feedback during imaging to control for possible habituation effects to feedback for well known face pairs. Nevertheless, the overall smaller activations observed in the interoceptive feedback condition may be partially explained by some satiety effects and changes in reward value across the duration of training.

\section{Cognitive implications}

The exact nature of the cognitive operations within VPFC remains undetermined. This region underlies encoding and flexible updating of stimulus-response associations (Rolls et al., 1994; Passingham et al., 2000; Nagahama et al., 2001). The temporal resolution of PET prevents more detailed study of timedependent VPFC activity. Our preliminary functional magnetic resonance imaging (MRI) block design studies suggest that a buildup of blood oxygenation level-dependent signal occurs in OPFC during training.

This study does not clarify the nature of the representation of feedback-dependent associations between stimulus and correct response. Although not the focus of this study, the same precuneus region activated during TEST across both studies. Thus, visual codes may be used in the hierarchy representation. Likewise, little is known about the representation of the relational operator (greater than, $>$ ) and its relationship to affective/motivational processes. Activity in the amygdaloid region, situated medially in periamygdaloid cortex/basal forebrain regions, varied along with the VMPFC across feedback types. Such activation is consistent with projections from medial aspects of the amygdala to hypothalamus, and midbrain, participating in the regulation of visceral functions (for review, see Price, 2003). Of note, subjects were neither thirsty nor hungry at the time of training or TEST. Therefore, the amygdaloid activity probably is not coding the valence of the feedback but more likely the integration of autonomic interoceptive processes related to the feedback.

The VTA/SN also activated during TEST after training with visceroappetitive feedback. Connections between VMPFC and VTA/SN are well established (Porrino et al., 1981; Öngür and Price, 2000; Tzschentke and Schmidt, 2000), and its role in feeding as part of the mesolimbic dopamine pathway has been identified 


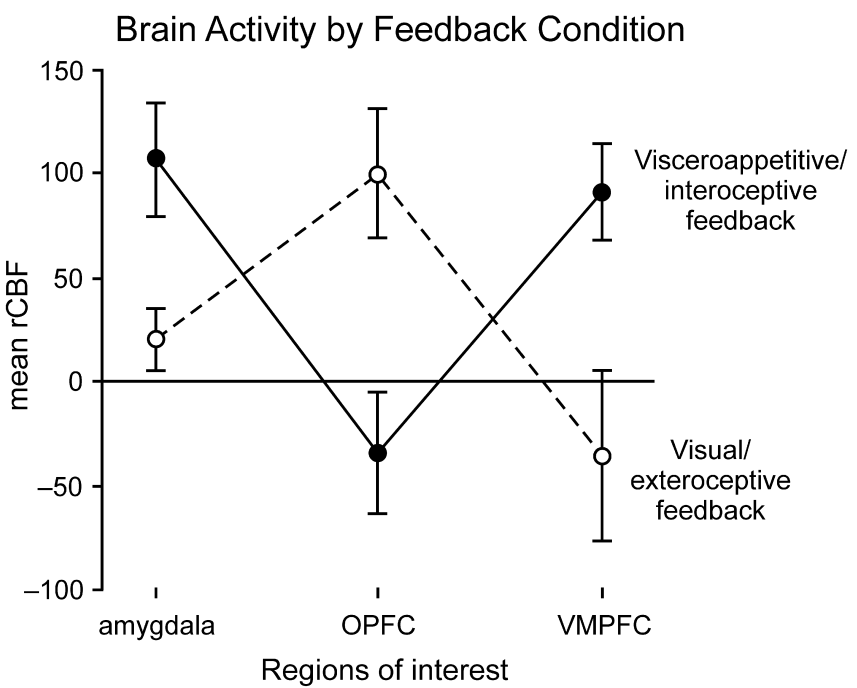

Figure 3. Region-of-interest analysis for the contrast between TEST and CONTROL for exteroceptive versus interoceptive feedback. Regions of interest were defined based on results from previous whole-brain group subtractions. (The OPFC region of interest was defined based on peak pixel activation in the visual/exteroceptive feedback group at $33 \mathrm{~mm}, 30 \mathrm{~mm},-18$ $\mathrm{mm}$; the VMPFC region of interest was defined based on peak pixel activation in the visceroappetitive/interoceptive feedback group at $-6 \mathrm{~mm}, 21 \mathrm{~mm},-16 \mathrm{~mm}$.) Mean rCBF calculations for both regions of interest were performed on a box including 27 nearest neighbor pixels from the peak pixel. Bars represent SEs of estimate.

(Korotkova et al., 2003; MacDonald et al., 2003). Recent neuroimaging studies are beginning to show that both VMPFC and VTA/SN are important in human food consumption and obesity (J. V. Pardo, S. A. Sheikh, B. R. Rittberg, and D. E. Adson, unpublished observation), consistent with a role in visceroappetitive/interoceptive processing. Stimulation of OPFC has multiple autonomic effects, suggesting that the VPFC is a major cortical representation of the vagus nerve (for review, see Fuster, 1989). Unfortunately, the resolution prevents more definitive identification of such structures activated during TEST.

How can fruit juice, a sensory stimulus, activate medial/interoceptive systems without activating lateral/exteroceptive systems? Typically, activations in primary and secondary association cortices for flavors, tastes, and smells are not observed with repeated stimulation (Small et al., 1999; Zald and Pardo, 2000a). As expected, we do not find primary or secondary taste cortex activations with the fruit juice stimulus delivered over $>1 \mathrm{~h}$. Thus, the observed activations do not represent the sensory/discriminative stimulus component but its intrinsic value.

Could a difference in rewarding properties between the two feedback types be the main reason for the different activations? A follow-up study that directly manipulated the "wanting" component of reward clarifies this. There, fasting subjects acquired the TI task with interoceptive feedback. All subjects were fed before PET scanning. In the follow-up, more anterior VMPFC activation emerges (fasting, $y=32 \mathrm{~mm}$; satiated, $y=21 \mathrm{~mm}$ ), consistent with other studies that observed anterior VMPFC activation with rewarding stimuli (Gottfried et al., 2003; Kringelbach and

Table 4. Region of interest by feedback condition covariate analysis (mixed model)

\begin{tabular}{lll}
\hline Region & $F$ test & $p$ value \\
\hline OPFC $\times$ feedback & $F_{(1,19)}=9.56$ & 0.006 \\
VMPFC $\times$ feedback & $F_{(1,19)}=7.20$ & 0.0147 \\
Extended amygdala $\times$ feedback & $F_{(1,19)}=7.23$ & 0.0145 \\
\hline
\end{tabular}

Regions of interest were defined based on results from previous whole-brain group subtractions.

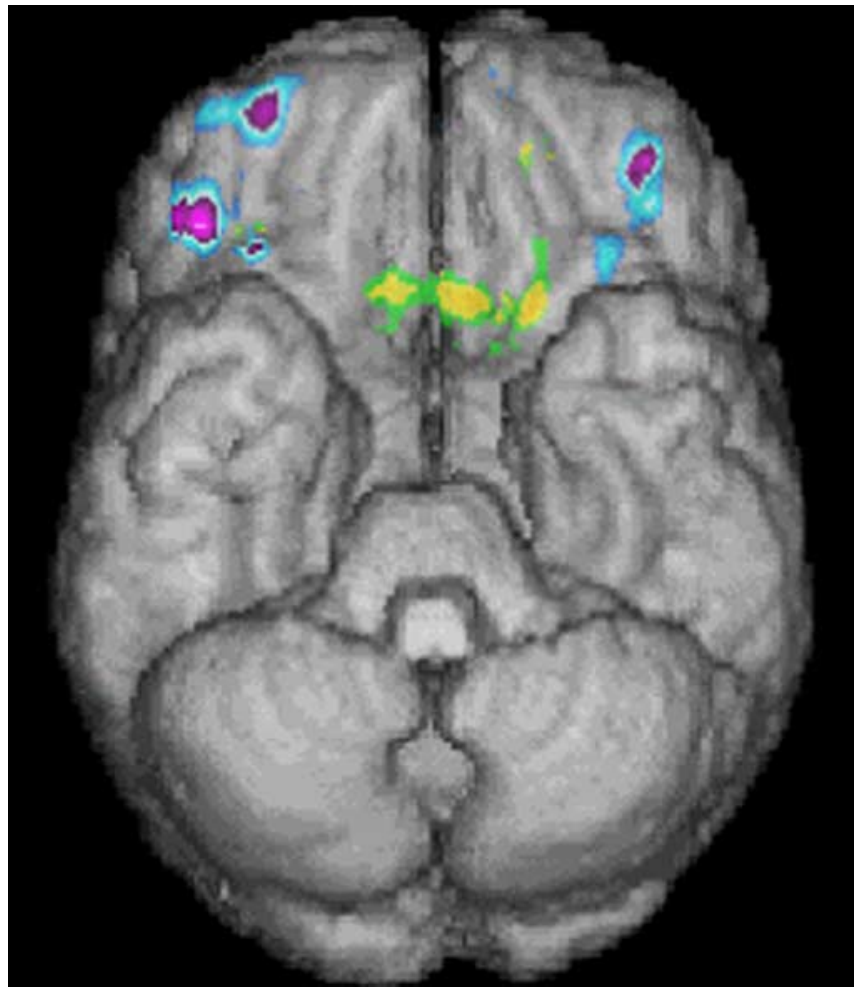

Figure 4. Region-of-interest analysis for the interaction between brain region and feedback group. Ventral prefrontal cortex regions of greater activation in the interoceptive feedback condition are displayed in the yellow-green color scale (Z score of 2.5). Ventral prefrontal cortex regions of greater activation in the exteroceptive feedback condition are displayed in the purple-blue color scale (Z score of 2.7).

Rolls, 2004). Furthermore, although we observe activations in $\mathrm{VTA} / \mathrm{SN}$, there is no clear consensus in the literature regarding the structures underlying aspects of reward processing ["wanting" vs "liking" (Wyvell and Berridge, 2000)]. In the follow-up experiment, we observe additional strong activations in anatomical regions typically associated with reward, such as ventral striatum (coordinates, $-21 \mathrm{~mm}, 14 \mathrm{~mm},-9 \mathrm{~mm} ;=4.5$ in a within-group comparison). It is possible that VTA/SN activations observed in the current study represent "liking" or the hedonic component of interoceptive stimuli.

VPFC activations in the current studies emerged only in a limited portion of this extensive frontal region. Functional specificity likely exists within VPFC. In addition to sensory association cortices for taste in the insula and anterior cingulate, the VPFC is known to participate in sensory taste processing (Kobayashi et al., 2002; Rolls et al., 2003; de Araujo et al., 2004). It also participates in the flexible updating of associations to adjust to new contingencies (Rolls et al., 1994; Freedman et al., 1998; Baxter et al., 2000). Furthermore, some VPFC region may be crucial in predicting reward value (Rolls et al., 1989; Tremblay and Schultz, 1999; O’Doherty et al., 2000), and neurons seem to discriminate between reward values (Rolls et al., 1996; Passingham et al., 2000; Gottfried et al., 2003).

The functional double dissociation within VPFC feedback subregions suggests that exteroceptive and interoceptive feedback cannot be processed equally at the same time. Inappropriate activation of the medial subsystem may lead to deactivation of the lateral subsystem at the expense of exteroceptive feedback processing. Limited capacity for dual processing could have profound implications for cognitive disorders with impaired deci- 

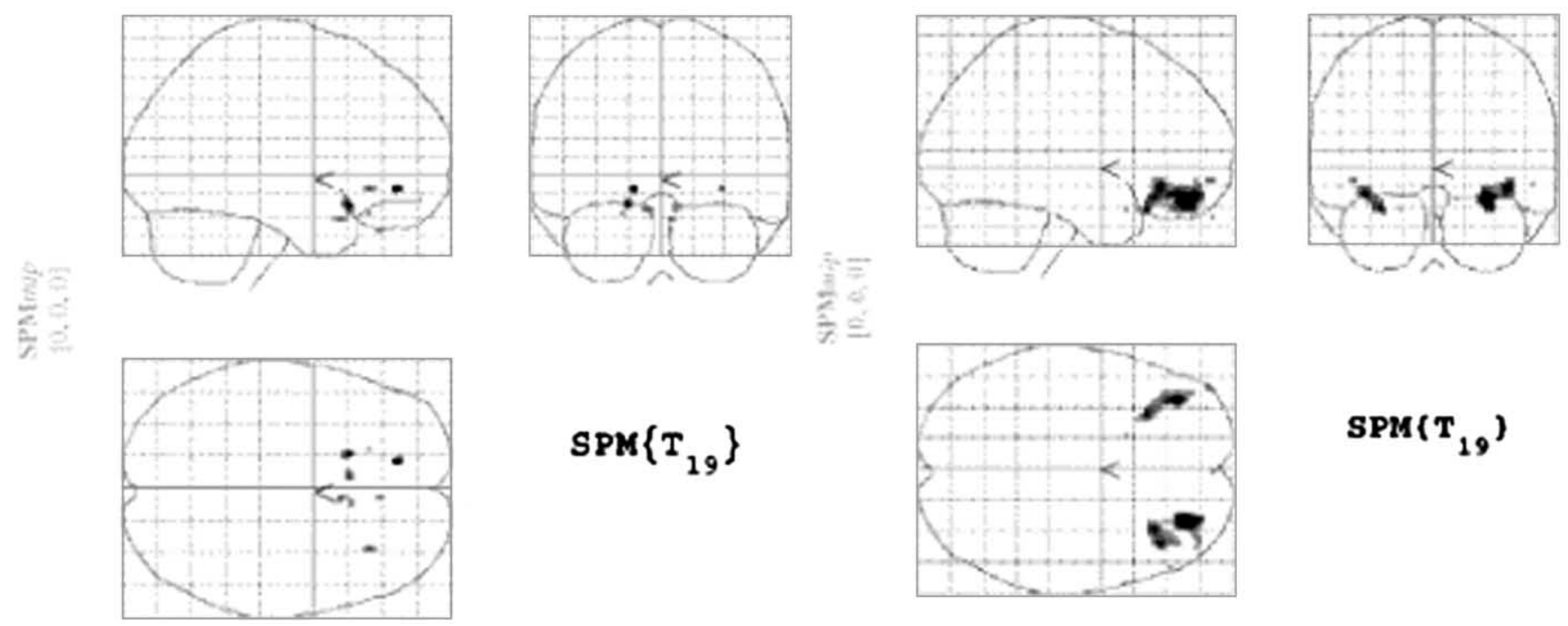

$\operatorname{SPM}\left(\mathbf{T}_{19}\right)$

Figure 5. Global brain analysis for interactions between feedback conditions and the ventral prefrontal regions of interest. Glass brain projections with ventral prefrontal activations for the subtraction TEST - CONTROL in visceral versus visual feedback (left; VPFC activations greater than Z score of 2.4) and for the subtraction TEST - CONTROL in visual versus visual feedback (right; VPFC activations greater than Z score of 2.3). SPM, Statistical Parametric Mapping.

sion making in general and addiction in particular. Overemphasis of interoceptive stimuli could lead to viscerally biased decisions that neglect external/cognitive signals. Such bias may allow addicts to neglect long-term negative social and occupational consequences associated with drug use in favor of immediate visceral rewards or relief of negative visceral stimuli. Paulus et al. (2002) showed that methamphetamine-dependent subjects failed to activate this region (BA 10/11) during a twochoice task. Bechara and Damasio (2002) found differences in a VMPFC-dependent gambling task in addicted subjects. Preliminary data of several long-term cocaine users showed that these volunteers inappropriately recruited the VMPFC and deactivated the OPFC when solving our cognitive activation task under the visual/exteroceptive feedback condition (our unpublished observation). Implications thus exist for psychopathological conditions with impairment in decision-making attributable to overemphasis of interoceptive stimuli (Porrino and Lyons, 2000; Goldstein and Volkow, 2002), because adaptive functioning relies partly on appropriate recruitment of VPFC feedback regions.

\section{References}

Barbas H, Rempel-Clower N (1997) Cortical structure predicts the pattern of corticocortical connections. Cereb Cortex 7:635-646.

Baxter MG, Parker A, Lindner CCC, Izquierdo AD, Murray EA (2000) Control of response selection by reinforcer value requires interaction of amygdala and orbital prefrontal cortex. J Neurosci 20:4311-4319.

Bechara A, Damasio H (2002) Decision-making and addiction. I. Impaired activation of somatic states in substance dependent individuals when pondering decisions with negative future consequences. Neuropsychologia 40:1675-1689.

Bechara A, Damasio H, Tranel D, Damasio AR (1997) Deciding advantageously before knowing the advantageous strategy. Science 275:1293-1295.

Bucholz KK, Robins LN, Shayka JJ, Przybeck TR, Helzer JE, Goldring E, Klein MH, Greist JH, Erdman HP, Skare SS (1991) Performance of two forms of a computer psychiatric screening interview: version I of the DISSI. J Psychiatr Res 25:117-129.

Cechetto DF, Chen SJ (1990) Subcortical sites mediating sympathetic responses from insular cortex in rats. Am J Physiol 258:245-255.

Chiavaras MM, LeGoualher G, Evans A, Petrides M (2001) Threedimensional probabilistic atlas of the human orbitofrontal sulci in standardized stereotaxic space. NeuroImage 13:479-496.

Craig AD (2002) How do you feel? Interoception: the sense of the physiological condition of the body. Nat Neurosci 3:655-666. de Araujo IET, Rolls ET, Kringelbach ML, McGlone F, Phillips N (2003) Taste-olfactory convergence, and the representation of the pleasantness of flavour, in the human brain. Eur J Neurosci 18:2059-2068.

Elliott R, Dolan RJ, Frith CD (2000) Dissociable functions in the medial and lateral orbitofrontal cortex: evidence from human neuroimaging studies. Cereb Cortex 10:308-317.

Freedman M, Black S, Ebert P, Binns M (1998) Orbitofrontal function, object alternation and perseveration. Cereb Cortex 8:18-27.

Fuster JM (1989) The prefrontal cortex: anatomy, physiology, and neuropsychology of the frontal lobe, Chap 2, pp 3-32. New York: Raven.

Goldstein RZ, Volkow ND (2002) Drug addiction and its underlying neurobiological basis: neuroimaging evidence for the involvement of the frontal cortex. Am J Psychiatry 159:1642-1652.

Gottfried JA, O’Doherty J, Dolan RJ (2003) Encoding predictive reward value in human amygdala and orbitofrontal cortex. Science 301:1104-1107.

Gusnard DA, Raichle ME (2001) Searching for a baseline: functional imaging and the resting brain. Nat Rev Neurosci 2:685-694.

Jänig W (1996) Neurobiology of visceral afferent neurons: neuroanatomy, functions, organ regulation and sensations. Biol Psychol 42:29-51.

Kobayashi M, Sasabe T, Takeda M, Kondo Y, Yoshikubo S, Imamura K, Onoe H, Sawada T, Watanabe Y (2002) Functional anatomy of chemical senses in the alert monkey revealed by positron emission tomography. Eur J Neurosci 16:975-980.

Korotkova TM, Sergeeva OA, Eriksson KS, Haas HL, Brown RE (2003) Excitation of ventral tegmental area dopaminergic and nondopaminergic neurons by orexins/hypocretins. J Neurosci 23:7-11.

Kringelbach ML, Rolls ET (2004) The functional neuroanatomy of the human orbitofrontal cortex: evidence from neuroimaging and neuropsychology. Prog Neurobiol 72:341-372.

Kuskowski M, Pardo JV (1999) The role of the fusiform gyrus in successful encoding of face stimuli. NeuroImage 9:599-610.

Lee JT, Pardo JV (2000) iiv: a Java-based internet image viewer. NeuroImage 11:S918.

MacDonald AF, Billington CJ, Levine AS (2003) Effects of the opioid antagonist naltrexone on feeding induced by DAMGO in the ventral tegmental area and in the nucleus accumbens shell region in the rat. Am J Physiol 285:R999-R1004.

Mazoyer B, Zago L, Mellet E, Bricogne S, Etard O, Houde O, Crivello F, Joliot M, Petit L, Tzourio-Mazoyer N (2001) Cortical networks for working memory and executive functions sustain the conscious resting state in man. Brain Res Bull 54:287-298.

Minoshima S, Koeppe RA, Frey KA, Kuhl DE (1994) Anatomic standardization: linear scaling and nonlinear warping of functional brain images. J Nucl Med 35:1528-1537.

Nagahama Y, Okada T, Katsumi Y, Hayashi T, Yamauchi H, Oyanagi C, 
Konishi J, Fukuyama H, Shibasaki H (2001) Dissociable mechanisms of attentional control within the human prefrontal cortex. Cereb Cortex 11:85-92.

Nagode JC, Pardo JV (2002) Human hippocampal activation during transitive inference. NeuroReport 13:939-944.

O’Doherty J, Rolls ET, Francis S, Bowtell R, McGlone F, Kobal G, Renner B, Ahne G (2000) Sensory-specific satiety-related olfactory activation of the human orbitofrontal cortex. NeuroReport 11:893-897.

Oldfield RC (1971) The assessment and analysis of handedness: The Edinburgh Inventory. Neuropsychologia 9:97-113.

Öngür D, Price JL (2000) The organization of networks within the orbital and medial prefrontal cortex of rats, monkeys and humans. Cereb Cortex 10:206-219.

Öngür D, Ferry AT, Price JL (2003) Architectonic subdivision of the human orbital and medial prefrontal cortex. J Comp Neurol 460:425-449.

Passingham RE, Toni I, Rushworth MFS (2000) Specialisation within the prefrontal cortex: the ventral prefrontal cortex and associative learning. Exp Brain Res 133:103-113.

Paulus MP, Hozack NE, Zauscher BE, Frank L, Brown GG, Braff DL, Schuckit MA (2002) Behavioral and functional neuroimaging evidence for prefrontal dysfunction in methamphetamine dependent subjects. Neuropsychopharmacology 26:53-63.

Porrino LJ, Lyons D (2000) Orbital and medial prefrontal cortex and psychostimulant abuse: studies in animal models. Cereb Cortex 10:326-333.

Porrino LJ, Crane AM, Goldman-Rakic P (1981) Direct and indirect pathways from the amygdala to the frontal lobe in rhesus monkeys. J Comp Neurol 198:121-136.

Price JL (2003) Comparative aspects of amygdala connectivity. In: The amygdala in brain function (Shinnick-Gallagher P, Pitkaenen A, Shekhar A, Cahill L, eds), pp 50-58. New York: The New York Academy of Sciences.

Rolls ET (1996) The orbitofrontal cortex. Philos Trans R Soc Lond B Biol Sci 351:1433-1443.

Rolls ET, Sienkiewicz ZJ, Yaxley S (1989) Hunger modulates the responses to gustatory stimuli of single neurons in the caudolateral orbitofrontal cortex of the macaque monkey. Eur J Neurosci 1:53-60.
Rolls ET, Hornak J, Wade D, McGrath J (1994) Emotion-related learning in patients with social and emotional changes associated with frontal lobe damage. J Neurol Neurosurg Psychiatry 57:1518-1524.

Rolls ET, Critchley HD, Mason R, Wakeman EA (1996) Orbitofrontal cortex neurons: role in olfactory and visual association learning. J Neurophysiol 75:1970-1981.

Rolls ET, Verhagen JV, Kadohisa M (2003) Representations of the texture of food in the primate orbitofrontal cortex: neurons responding to viscosity, grittiness, and capsaicin. J Neurophysiol 90:3711-3724.

Rushworth MF, Nixon PD, Eacott MJ, Passingham RE (1997) Ventral prefrontal cortex is not essential for working memory. J Neurosci 17:4829-4838

Small DM, Zald DH, Jones-Gotman M, Pardo JV, Zatorre R, Frey S, Petrides M (1999) Human cortical gustatory areas: a review of functional neuroimaging data. NeuroReport 10:7-14.

Talairach J, Tournoux P (1988) Co-planar stereotaxic atlas of the human brain. New York: Thieme.

Tremblay L, Schultz W (1999) Relative reward preference in primate orbitofrontal cortex. Nature 398:704-708.

Tzschentke TM, Schmidt WJ (2000) Functional relationship among medial prefrontal cortex, nucleus accumbens, and ventral tegmental area in locomotion and reward. Crit Rev Neurobiol 14:131-142.

Vaitl D (1996) Interoception. Biol Psychol 42:1-27.

Winstanley CA, Theobald DEH, Cardinal RN, Robbins TW (2004) Contrasting roles of basolateral amygdala and orbitofrontal cortex in impulsive choice. J Neurosci 24:4718-4722.

Wyvell CL, Berridge KC (2000) Intra-accumbens amphetamine increases the conditioned incentive salience of sucrose reward: enhancement of reward "wanting" without enhanced "liking" or response reinforcement. J Neurosci 20:8122-8130.

Zald DH, Pardo J (2000a) Functional neuroimaging of the olfactory system in humans. Int J Psychophysiol 36:165-181.

Zald DH, Pardo JV (2000b) Cortical activation induced by intraoral stimulation with water in humans. Chem Senses 25:267-275. 